\title{
Forced Expiratory Volume in 1 Second Reversibility
}

National Cancer Institute

\section{Source}

National Cancer Institute. Forced Expiratory Volume in 1 Second Reversibility. NCI

Thesaurus. Code C112285.

The change in FEV1 following administration of a bronchodilator relative to the pretreatment FEV1 value. (CDISC) 